\title{
EFECTO DE LA CONCENTRACIÓN DE RESIDUOS CERÁMICOS ODONTOLÓGICOS EN LAS PROPIEDADES TERMOFÍSICAS DE MATERIALES COMPUESTOS A BASE DE RESINAS DE POLIÉSTER
}

\author{
(Effect of ceramic dental waste in thermo-physical properties of materials composed with polyester resins)
}

\section{Lorena Martínez Maldonado*, Gabriel Peña Rodríguez **}

\begin{abstract}
* Facultad de Ingenierías. Plan de Estudios de Ingeniería Industrial. Semillero de Investigación en Materiales Cerámicos (SIMAC). Universidad Francisco de Paula Santander. Cúcuta, Colombia. lore 3612@hotmail.com

**Facultad de Ciencias Básicas. Departamento de Física. Grupo GITEC. Universidad Francisco de Paula Santander. Cúcuta, Colombia gpenaro@ufps.edu.co
\end{abstract}

(Recibido: el 5 de Agosto de 2014 y aceptado 1 de Octubre de 2014)

\section{Resumen:}

Se reportan las propiedades termofísicas a temperatura ambiente de un material compuesto a base de resinas de poliéster y polvos obtenidos de los residuos cerámicos odontológicos, para mezclas con porcentaje en peso de $50-50,60-40,70-30,80-20$ y 90-10, donde la fase minoritaria son los polvos cerámicos odontológicos con tamaño de partícula pasante tamiz No. $200(75 \mu \mathrm{m})$, y la mayoritaria, resina de poliéster preacelerada referencia P-2000, y como catalizador (Meck-Peróxido). El proceso de elaboración de las probetas fue por colado en moldes cilíndricos de diámetro $3 \mathrm{~cm}$ y altura $6 \mathrm{~cm}$. Las propiedades de conductividad $(\mathrm{k})$ y difusividad $(\alpha)$ térmica, así como el calor específico por unidad de volumen $(\rho c)$, fueron hallados usando el sistema KD2 Pro ${ }^{\circledR}$, el cual funciona con el principio físico de flujo lineal transitorio de calor. La efusividad térmica $(\varepsilon)$ se determinó usando los datos de $\mathrm{k}$ y $\alpha$, y la expresión $\varepsilon=\mathrm{k}$ ? $\sqrt{ }$ $\alpha$. Los resultados muestran que a medida que se incrementa el porcentaje de los polvos cerámicos, la densidad de las muestras aumenta, y por ende la conductividad térmica $(\mathrm{k})$, la cual es directamente proporcional tanto a la rapidez de difusión de calor $(\alpha)$ como a la cantidad de calor que puede almacenar o liberar ( $\rho$ c) el material. Estos resultados proponen un nuevo material para aplicaciones tecnológicas, al igual que permiten contribuir a mitigar el impacto ambiental, debido al proceso de reciclaje de los residuos cerámicos odontológicos.

Palabras clave: flujo lineal transitorio de calor, materiales compuestos, propiedades termofísicas, residuos cerámicos odontológicos.

\section{INTRODUCCIÓN}

La gran demanda de nuevas tecnologías ha dado lugar a que los actuales procesos de manufactura requieran de nuevos materiales, ya sea en busca de una reducción de costos o a una optimización en el funcionamiento de los productos (Manzano, 2010). De esta manera, los materiales compuestos han adquirido una gran importancia a nivel mundial.

\section{Abstract:}

Thermophysical properties at room temperature of a composite material based on polyester resins and powders obtained from dental ceramic residues for mixtures with percentage by weight of 50-50, 60-40, 70$30,80-20$ and $90-10$ are recorded, where the minority phase are dental ceramic powders with particle size through sieve No. 200 (75 um), and the majority of preaccelerated polyester resin brand P-2000, and as catalyst (Meck-Peroxide). The manufacturing process of the specimens was by casting them into cylindrical molds of diameter $3 \mathrm{~cm}$ and $6 \mathrm{~cm}$ long. The properties of conductivity $(\mathrm{k})$ and thermal diffusivity $(\alpha)$ and the specific heat per unit volume $(\rho c)$, were found using the KD2 Pro ${ }^{\circledR}$ system which operates on the physical principle of linear transient heat flow. Thermal effusivity (ع) was determined using data from $\mathrm{k}$ and $\alpha$, and the expression $\varepsilon=\mathrm{k} / \sqrt{ } \alpha$. The results show that as the percentage of the ceramic powder is increased, the density of the samples increases, and thus the thermal conductivity $(\mathrm{k})$, which is directly proportional to both heat diffusion rate $(\alpha)$ and the amount of heat that the material can store or release $(\rho c)$. These results suggest a new material for technological applications, as well as they help to mitigate the environmental impact due to the recycling process of dental ceramic waste.
Keywords: linear transient heat flux, composites, thermophysical properties, dental ceramic waste.
El reto actual de los materiales compuestos consiste en buscar mejores asociaciones de matriz-refuerzo (Besednjak, 2011). Los compuestos termoestables conformados a partir de resinas curadas con catalizador, son de especial interés por su fácil manejo y conformación. En los últimos años han sido reforzados en su mayoría con fibras de vidrio, fique, polvos cerámicos y polvos de maíz, como respuesta a los retos que plantea la industria global (Elizetxea, Gurmendi \& Pijoan, 2010). 
Estos refuerzos se pueden obtener de productos reciclados, como lo plantea la filosofía de la ecología industrial, donde se pueden producir materiales para nuevas aplicaciones, con desechos o residuos, a fin de encontrar nuevas alternativas para promover el desarrollo sostenible de una región o país (Torre, Sosa, Rodríguez \& Robles, 2009, p. 65-67).

Las propiedades térmicas están íntimamente ligadas a los procesos térmicos (Artica, 2011), su importancia radica en que permiten conocer las respuestas térmicas de los materiales (Universidad del País Vasco, 2012), lo cual es de especial interés para establecer criterios de selección de los mismos para aplicaciones donde exista flujo de calor a través de estos.

Por lo anterior, en este trabajo se reportan las propiedades termofísicas de la conductividad (k), la cual expresa la capacidad de un material para conducir el calor, y es inherente a cada material (Ostachuk, Di Paolo \& Ulises, 2000 , p. 2-4). La difusividad ( $\alpha$ ), que es una medida de la cantidad de calor difundida a través de un material en calentamiento o enfriamiento en un tiempo determinado (Muñoz, 2002, p. 12-15), y la efusividad térmica $(\varepsilon)$, que indica la capacidad de los materiales para acumular calor (Domingo, 2012, p. 28-30), así como el calor especifico por unidad de volumen $(\rho c)$, es decir la cantidad de energía, en forma de calor, que gana o pierde un sistema (Orrego, 2003, p. 61-63) por unidad de volumen. Las medidas se realizaron a temperatura ambiente, en un material compuesto de matriz resina de poliéster termoestable, reforzado con polvos cerámicos reciclados de la industria odontológica.

\section{MATERIALES Y MÉTODOS}

Los residuos odontológicos que se emplearon fueron de dos clases: alginatos, los cuales son sales solubles del ácido alginico que se obtienen de las algas marinas llamadas alginas, compuestos también de diatomitas glicoles y antimicrobianos (dentaltvweb, 2013) y yeso dental tipo III o sulfato de calcio hemihidrato (López \& Alarcón, 2011, p. 138-139).

Los polvos cerámicos utilizados fueron obtenidos de los moldes dentales de yeso y alginato desechados en odontologías (ver Figura 1a y 1b), los cuales se secaron en una estufa de circulación forzada a.

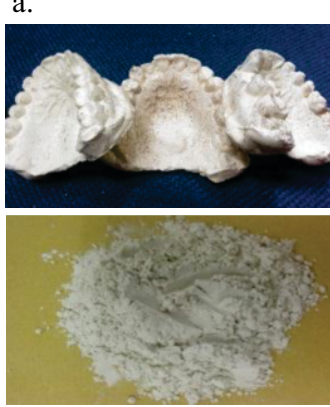

c.

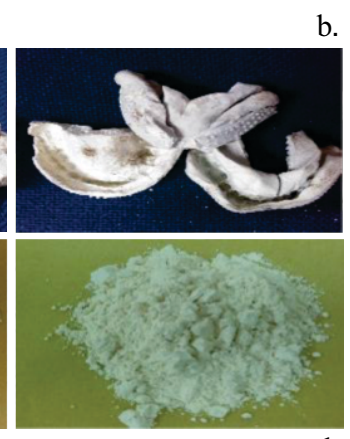

d.
Figura 1. Moldes recolectados. a) Moldes de yeso. b) Moldes de alginato. c) Polvos de yeso. d) Polvos de alginato
(Gabrielli) durante 24 horas a una temperatura de $1105^{\circ} \mathrm{C}$. Estos se trituraron manualmente con una mazo metálico y posteriormente se pulverizaron usando un molino de martillo marca Servitech con una potencia de $3 \mathrm{HP}$, luego se tamizaron en un tamiz marca Gran Test malla $200(75 \mu \mathrm{m})$, de este proceso se seleccionó el pasante.

La resina comercial empleada para las mezclas es de tipo Ortoftaltica insaturada preacelerada referencia P2000, distribuida por Industrias de Resinas S.A.S. Este tipo de resina es de uso general en ambientes no agresivos, de color rosado, contiene aproximadamente un $65 \%$ de poliéster. Para el proceso de sinterización se usó como catalizador MeckPeróxido en concentración de $4 \%$ en peso.

En la Tabla 1, se presentan las cinco mezclas seleccionadas para elaborar las probetas en porcentajes en peso, donde la fase mayoritaria fue la resina y la minoritaria fue una mezcla en partes iguales de los polvos de yeso y alginatos reciclados. El tiempo de mezclado de la resina y los polvos se estableció de forma constante por un lapso de diez minutos.

Tabla 1. Mezclas seleccionadas con porcentajes en peso.

\begin{tabular}{|c|c|}
\hline Porcentaje resina & $\begin{array}{c}\text { Porcentaje mezcla de } \\
\text { residuos }\end{array}$ \\
\hline 50 & 25 \\
\hline 60 & 20 \\
\hline 70 & 15 \\
\hline 80 & 10 \\
\hline 90 & 5 \\
\hline
\end{tabular}

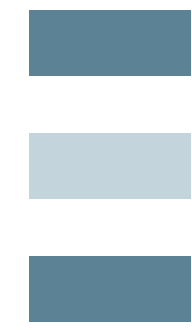

La medida de las propiedades termofísicas se realizó utilizando el sistema KD2 Pro ${ }^{\circledR}$ the dual needle SH-1 sensor, el cual se basa en el principio del flujo transitorio lineal de calor usando dos agujas o sensores de forma paralela, una aguja transmite el calor y la otra es una termocupla. Para lograr dar la forma cilíndrica a la probeta, como lo plantea el sistema, se cortaron tubos de PVC de 2" de diámetro con una altura de $120 \mathrm{~mm}$, y para conseguir que la probeta tuviera los dos agujeros para los sensores, se usaron agujas hipodérmicas de $60 \mathrm{~mm}$ largo y $0,82 \mathrm{~mm}$ de diámetro.

El modelo físico detrás de este método es la fuente de línea infinita con potencia constante por unidad de longitud, es decir, considera que si el calor a una velocidad constante $q$, se aplica a una fuente de línea infinitamente larga e infinitamente pequeña, la respuesta de la temperatura de la fuente, con el tiempo, puede ser descrita por la ecuación (Fontana, Varith, Ikediala, Reves \& Wacker, 1999, p. 2-4):

$$
K=\frac{q\left(\ln t \_2-\ln t_{-} 1\right)}{4 p\left(? T_{-} 2-T_{-} 1\right)}
$$

La medida de las propiedades termofísicas se realizó con repeticiones de cinco, con lapsos de cinco minutos entre medida y medida. En la Figura 2 se puede observar el proceso de medición de las propiedades termofísicas en una probeta.

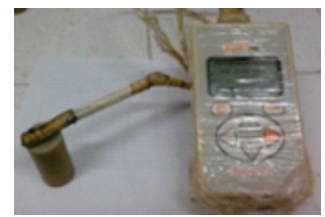

Figura 2. Toma de medidas de propiedades termofísicas con el sistema KD2 Pro ${ }^{\varpi}$.
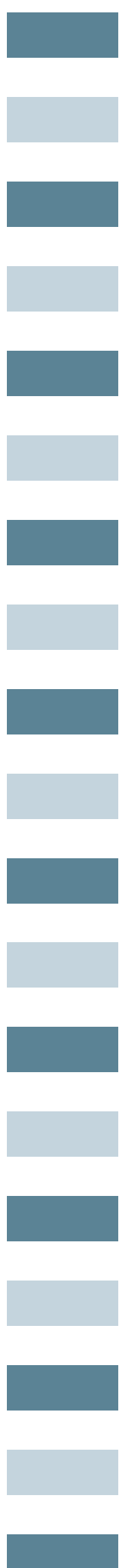

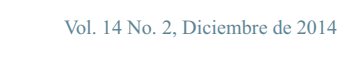




\section{RESULTADOS}

La Figura 3 está determinada por cuatro ilustraciones en las que se pueden apreciar las microfotografías realizadas con microscopía electrónica de barrido a los polvos de alginato y yeso reciclados, a una magnificación de $1.000 \mathrm{X}$ y $10.000 \mathrm{X}$. El equipo empleado es de referencia Quanta FEG 650.

De la Figura 3a y $3 b$ se puede inferir que la morfología de los polvos de alginato no es uniforme, pues se reporta la presencia de partículas en forma circular con diámetros entre los 15,4 y 17,06 micrómetros, y con pequeños poros en su contorno, de diámetros entre los 0,21 y 0,44 micrómetros. Como lo reportan Lemes et al. (2010), estas partículas corresponden a algas de la división Chrysophyta, clase Bacillariophycea. Los miembros de esta clase, conocidos como diatomeas, son esencialmente unicelulares y hacen parte de composición inorgánica del alginato (Lemes et al., 2010).

De la Figura 3c y 3 b se puede apreciar que la morfología de los polvos de yeso es irregular, constituida de partículas muy finas de diferentes geometrías.
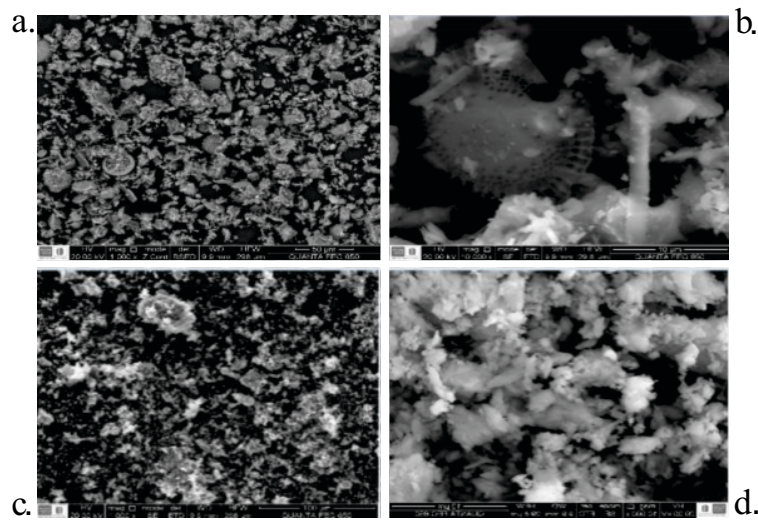

Figura 3. Microfotografías de los polvos de Residuos Odontológicos. a) Polvos de alginato a una magnificación de $1000 \mathrm{X}$. b) Polvos de alginato a una magnificación de 10000X. c) Polvos de yeso a una magnificación de 1000X. d) Polvos de yeso a una magnificación de $10000 \mathrm{X}$.

En la Figura 4 se puede observar la composición química de los polvos de yeso y alginato recuperados, resultado del microanálisis realizado por espectroscopia de energía dispersada (EDS) con una sonda EDAX serie SDD Apollo X y una corrección ZAF a dicho espectro.

La Figura 4a muestra la composición química de los polvos de alginato reciclados, con lo que se puede deducir que es un alginato sódico, conformado en su mayoría por oxígeno, sílice y carbono. De acuerdo como lo describen Bermúdez, Flórez y Cardona (2009) y en concordancia con lo analizado del espectro, otros de los componentes de menor proporción son azufre, calcio, potasio y zinc.

De acuerdo con la Figura 4b, la cual corresponde a los polvos de yeso recuperado, los principales componentes son calcio, oxígeno y azufre, como lo reportan Li-Hong et al. (2010).
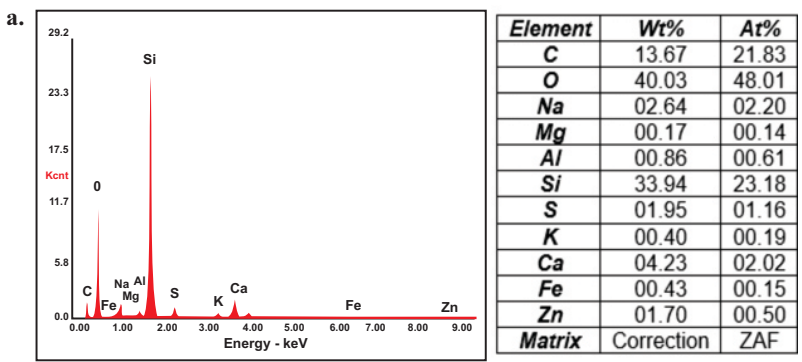

b.

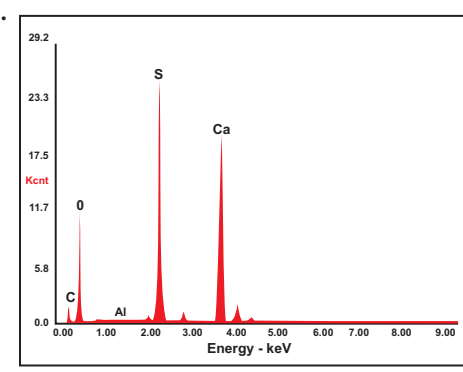

\begin{tabular}{|c|c|c|}
\hline Element & $\boldsymbol{W t} \%$ & $\boldsymbol{A t} \%$ \\
\hline $\mathbf{C}$ & 13.67 & 21.83 \\
\hline $\mathbf{O}$ & 40.03 & 48.01 \\
\hline $\mathbf{N a}$ & 02.64 & 02.20 \\
\hline $\mathbf{M g}$ & 00.17 & 00.14 \\
\hline $\mathbf{A l}$ & 00.86 & 00.61 \\
\hline $\mathbf{S i}$ & 33.94 & 23.18 \\
\hline $\boldsymbol{S}$ & 01.95 & 01.16 \\
\hline $\boldsymbol{K}$ & 00.40 & 00.19 \\
\hline $\mathbf{C a}$ & 04.23 & 02.02 \\
\hline $\mathbf{F e}$ & 00.43 & 00.15 \\
\hline $\mathbf{Z n}$ & 01.70 & 00.50 \\
\hline Matrix & Correction & ZAF \\
\hline
\end{tabular}

Figura 4. EDX y composición química de los polvos reciclados. a) Polvos de alginato. b) Polvos de yeso.

Tabla 2. Propiedades termofísicas del compuesto

\begin{tabular}{|c|c|c|c|c|c|}
\hline MUESTRA & $\begin{array}{c}\rho\left(\mathrm{Kg}^{3} / \mathrm{m}^{3}\right) \\
\mathrm{X} 10^{3}\end{array}$ & $\mathrm{~K}\left(\mathrm{~W} / \mathrm{m}^{\circ} \mathrm{C}\right)$ & $\begin{array}{c}\alpha\left(\mathrm{m}^{2} / \mathrm{s}\right) \\
\mathrm{x} 10^{-7}\end{array}$ & $\begin{array}{c}\rho \mathrm{c}\left(\mathrm{J} / \mathrm{m}^{3 \circ} \mathrm{C}\right) \\
\mathrm{x} 10^{6}\end{array}$ & $\begin{array}{c}\varepsilon=\mathrm{k} / \mathrm{V} \\
\left(\mathrm{Ws}^{1 / 2} / \mathrm{m}^{2}{ }^{\circ} \mathrm{C}\right)\end{array}$ \\
\hline 100 & $1,198 \pm 55,19$ & $0,1635 \pm 0,001$ & $0,96 \pm 0,00$ & $1,699 \pm 0,007$ & $527,694 \pm 3,23$ \\
\hline $90-10$ & $1,295 \pm 48,95$ & $0,1887 \pm 0,004$ & $1,31 \pm 0,02$ & $1,443 \pm 0,042$ & $521,496 \pm 7,07$ \\
\hline $80-20$ & $1,341 \pm 54,29$ & $0,2132 \pm 0,009$ & $1,39 \pm 0,05$ & $1,54 \pm 0,107$ & $571,981 \pm 13,85$ \\
\hline $70-30$ & $1,421 \pm 41,57$ & $0,2506 \pm 0,016$ & $1,66 \pm 0,01$ & $1,508 \pm 0,083$ & $615,135 \pm 37,42$ \\
\hline $60-40$ & $1,512 \pm 89,26$ & $0,2810 \pm 0,006$ & $1,68 \pm 0,05$ & $1,59 \pm 0,057$ & $652,938 \pm 9,61$ \\
\hline $50-50$ & $1,517 \pm 70,32$ & $0,3105 \pm 0,004$ & $1,95 \pm 0,01$ & $1,592 \pm 0,016$ & $703,144 \pm 7,26$ \\
\hline
\end{tabular}

En la Tabla 2 se reportan las propiedades termofísicas del compuesto $(\mathrm{K}, \alpha, \rho \mathrm{c}, \varepsilon)$, las cuales se midieron a temperatura ambiente con el sistema KD2 Pro. La efusividad se determinó usando los datos de $\mathrm{k}$ y $\alpha$, y la expresión $\varepsilon=k$ ? $\sqrt{ } \alpha$. De la misma forma se presenta el valor de la densidad ( $\rho$ ) para cada mezcla elaborada, como unidad de masa sobre volumen.

El valor de $\mathrm{K}$ de la resina de poliéster insaturada según Miravete (2007), se encuentra en el rango de 0,11 y $0,28 \mathrm{~W} / \mathrm{m}$ ?C. De la misma forma, el Prontuario de Soluciones Constructivas (2007) plantea un valor de $0.19 \mathrm{~W} / \mathrm{m}$ ? C para $\mathrm{k}$, de $1,68 \times 10^{6} \mathrm{~J} / \mathrm{m}^{3}$ ? C para $\rho c$ y de $1400 \mathrm{Kg} / \mathrm{m} 3$ para la densidad, lo cual va en concordancia con los datos reportados en la Tabla 2 y genera confiabilidad en los resultados presentados.

En la Figura 5 se grafica $K, \alpha, \rho c$ para cada una de las mezclas. En esta figura se puede apreciar que, a medida que se incrementa la concentración de polvos, aumentan $\mathrm{K}$ y $\alpha$.

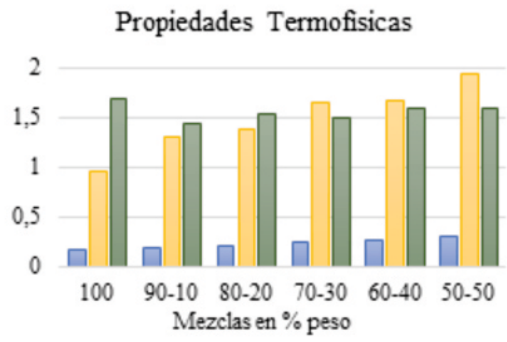

Figura 5. Propiedades térmicas para las diferentes mezclas $\mathrm{K}\left(\mathrm{W} / \mathrm{m}^{\circ} \mathrm{C}\right) \square \alpha\left(\mathrm{m}^{2} / \mathrm{s}\right) \times 10^{-7} \square \rho c\left(\mathrm{~J} / \mathrm{m}^{3 \circ} \mathrm{C}\right) \times 10^{6}$ 
La línea roja de la Figura 6 muestra la relación entre $\rho$ y K, la cual fue ajustada con una función cuadrática. En esta se puede observar una tendencia de crecimiento entre las dos propiedades, dicho comportamiento es similar al reportado por Gonzales (2011, p. 3-5) para materiales de construcción. De la Figura 6 se puede deducir que al incrementarse la $\rho$ del compuesto, la $\mathrm{K}$ aumenta, lo cual es de esperarse, ya que al ser más denso el material, menos aire tendrá en su interior, lo cual permitirá que el flujo de calor sea mayor (Gonzales, 20XX, p. 3-5).

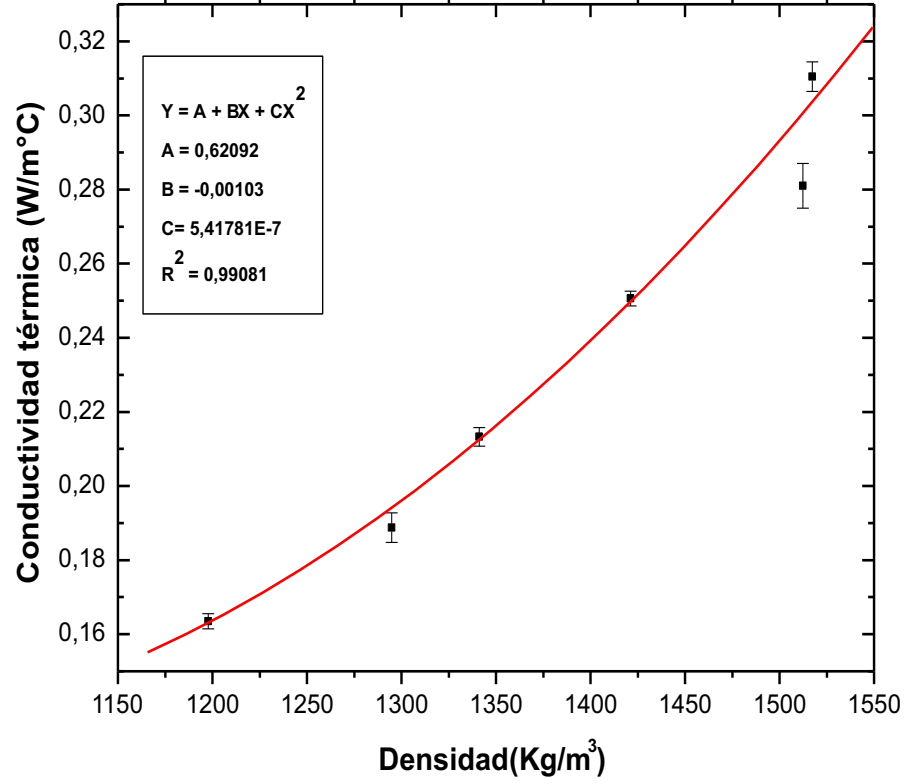

Figura 6. Relación entre K y $\rho$ de las diferentes mezclas del compuesto.

\section{CONCLUSIONES}

Se reporta la $\rho$, la $K$, la $\alpha$, el $\rho$ c y la ? a temperatura ambiente, con diferentes mezclas de un material compuesto de matriz resina de poliéster y refuerzo de polvos de yeso y alginato, obtenidos de residuos de la industria odontológica. De lo anterior se concluye que al aumentar la concentración de polvos, se incrementan K y $\alpha$, de igual forma se presenta una relación creciente entre $\mathrm{K}$ y $\rho$.

Los valores de $\mathrm{K}$ del polietileno de baja densidad, de un bloque cerámico, del fibrocemento y del aplanado de yeso, son muy cercanos al reportado para la mezcla de 50-50\%. Los compuestos elaborados con las diferentes mezclas poseen muy baja K, pero no pueden ser considerados como aislantes. De igual forma, los valores de la $\alpha$ de las mezclas reforzadas, son muy similares a las reportadas en la literatura para las maderas de pino y de alerce.

\section{AGRADECIMIENTOS}

Al Fondo de Investigaciones Universitarias FINU- UFPS, por el apoyo económico recibido mediante contrato 041-2013.

A la Clínica Odontológica Sonricenter, por facilitar los moldes de yeso y alginato.

\section{REFERENCIAS}

Artica L. (2011). Cálculo de propiedades termofisicas. Perú: Universidad Nacional del Centro del Perú, Facultad de ingeniería en Industrias Alimentarias.

Bermúdez, C., Flórez I. \& Cardona, L. (2009). Manual de procedimientos para el montaje y análisis de modelos articulados. Medellín: Universidad CES, Facultad de Odontología.
Dentalweb (2013). Alginato dental. Recuperado de http://dentaltvweb.com/blog/?p=123

Domingo, J. (2012). Evaluación del comportamiento térmico e higrotérmico de cerramientos de madera. Trabajo de Maestría. Pamplona: Universidad de Navarra.

Elizetxea, C., Gurmendi, U. \& Pijoan, T. (2010). Composites con refuerzos naturales para aplicaciones en automoción y construcción. Revista de metalurgia, 46, 138-139.

Fontana A., Varith J., Ikediala, J., Reves J. \& Wacker, B. (1999). Thermal properties of selected foods using a dual-needle heat-pulse sensor. En: Institute of Food Technologists Annual Meeting; July 24-28 Chicago; p. 2-4.

Gonzales E. (2010). Selección de materiales en la concepción arquitectónica bioclimática. Maracaibo: Instituto de Investigaciones de la Facultad de Arquitectura y Diseño.

Lemes, C., Borges, R., Souza, L., Correr, L., Soares, C. \& Coelho, S. (2010). Analysis of filler

particle levels and sizes in dental alginates. Mat. Res, 13(2), 310

Li-Hong, H., Vuuren, L., Planitz, N. \& Swain, M. (2010). A micromechanical evaluation of the effects of die hardener on die stone. Dental Materials Journal, 29(4), 433-437.

López, J. \& Alarcón, M. (2011). Sulfato de calcio: propiedades y aplicaciones clínicas. Revista Clínica. Periodoncia Implantol, Rehabilitación Oral, 4(3), 138-139.

Manzano, A. (2010). Materiales compuestos, los materiales del siglo XXI. Querétaro: Centro de Investigación y de estudios Avanzados del IPN.

Miravete, A. (2007). Materiales compuestos I. Vol. 1. Barcelona: Reverté.

Muñoz J. (2002). Determinación de la difusividad térmica en Pasta de Murta (Ugni molinae Turcz), en función de la temperatura. Proyecto de grado. Valdivia: Universidad Austral de Chile, Facultad de Ciencias Agrarias, Escuela de Ingeniería en Alimentos.

Orrego, C. (2003). Capítulo 3, Propiedades físicas de los alimentos. En Procesamiento de alimentos. (pp. 61-63). Universidad Nacional de Colombia sede Manizales.

Ostachuk, A., Di Paolo, L. \& Ulises, O. (2000). Una manera simple de determinar la conductividad térmica de los materiales. San Martín, Argentina: Universidad Nacional de General San Martín.

Prontuario de Soluciones Constructivas. (2007). Recuperado de http://cte-web.iccl.es/materiales.php?a $=16$

Torre, C., Sosa, G., Rodríguez, R. \& Robles, F. (2009). Ecología industrial y desarrollo sustentable. Revista de Ingeniería, 13, 65-67.

Universidad del País Vasco, (2012). Propiedades térmicas. Capítulo 19.
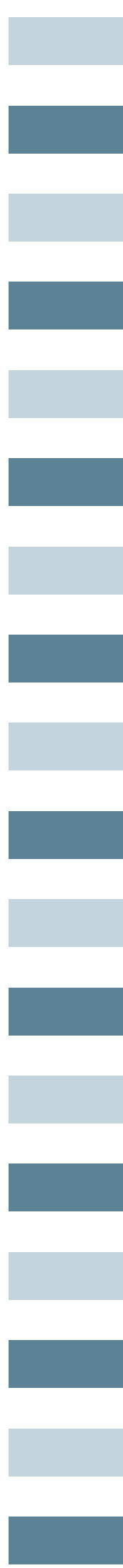

$$
\text { Capitulo } 19 .
$$

